\title{
Genetic variation in subdivided populations and conservation genetics
}

\author{
Sirkka-Liisa Varvio*, \\ Ranajit Chakraborty and Masatoshi Nei
}

\author{
University of Texas Health Science Center at \\ Houston, Center for Demographic and Population \\ Genetics, P.O. Box 20334, Houston, Texas 77225
}

The genetic differentiation of populations is usually studied by using the equilibrium theory of Wright's infinite island model. In practice, however, populations are not always in equilibrium, and the number of subpopulations is often very small. To get some insight into the dynamics of genetic differentiation of these populations, numerical computations are conducted about the expected gene diversities within and between subpopulations by using the finite island model. It is shown that the equilibrium values of gene diversities $\left(H_{S}\right.$ and $\left.H_{T}\right)$ and the coefficient of genetic differentiation $\left(G_{S T}\right)$ depend on the pattern of population subdivision as well as on migration and that the $G_{S T}$ value is always smaller than that for the infinite island model. When the number of migrants per subpopulation per generation is greater than 1, the equilibrium values of $H_{S}$ and $H_{T}$ are close to those for panmictic populations, as noted by previous authors. However, the values of $H_{S}, H_{T}$, and $G_{S T}$ in transient populations depend on the pattern of population subdivision, and it may take a long time for them to reach the 95 per cent range of the equilibrium values. The implications of the results obtained for the conservation of genetic variability in small populations are discussed. It is argued that any single principle should not be imposed as a general guideline for the management of small populations.

\section{INTRODUCTION}

Theoretical studies of genetic differentiation of subpopulations have shown that, in the absence of selection, the migration rate among subpopulations must be very small for the differentiation to be significantly large (Wright, 1951; Maruyama, 1970; Kimura and Maruyama, 1971; Nei and Feldman, 1972). This theoretical conclusion has been applied to the problem of management of small endangered populations. Thus, it has been suggested that the exchange of a single individual per generation among zoo, or other small, populations is sufficient to make them behave almost as one panmictic population (Franklin, 1980; Frankel and Soulé, 1981; Ralls et al., 1983; Frankel, 1983; Chambers, 1983; Allendorf, 1983). Maintenance of several small populations rather than a single large population has a number of ecological advantages. Therefore, if the genetic drawbacks of small populations, such as depletion of genetic variability and inbreeding depression, can be avoided by

\footnotetext{
* Present address: Department of Genetics, University of Helsinki, Arkadiankatu 7, SF-00100 Helsinki, Finland.
}

the exchange of one or two individuals per generation, this management procedure has a great advantage.

However, there are at least two problems in this argument. First, although the genetic differentiation as measured by $G_{S T}(\mathrm{Nei}, 1973)$ may not increase very much under this condition, the homozygosity within each subpopulation would increase if the effective population size is small, and this would in turn cause inbreeding depression. [We use the notation $G_{S T}$ rather than Wright's (1951) $F_{S T}$ because we consider the ratio of interpopulational genetic variation to the total variation.] The amount of differentiation measured by $G_{S T}$ is independent of the level of gene diversity, and it should be noted that the maintenance of genetic variability in the total population and the inbreeding depression in the subpopulations are separate problems. The former can be studied by using the gene diversity (heterozygosity) for the entire population $\left(H_{T}\right)$, whereas the latter should be studied by using the average gene diversity for subpopulations $\left(H_{S}\right)$. Second, the principle of one migrant per generation has been derived from studies of the steady-state behaviour of $G_{S T}$, and 
it usually takes a long time for the population to reach the equilibrium state. In the management of endangered populations, however, we are mainly concerned with the loss of genetic variability and inbreeding depression in the early generations. Therefore, the principle of one migrant may not be realistic.

The purpose of this paper is to examine the above two problems by using the finite island model studied by Maruyama (1970), Nei (1975), and $\mathrm{Li}$ (1976). Since the mathematical theory for the finite island model has been worked out, our task is to do several numerical computations to study our problems. We shall first investigate the relationship among the equilibrium values of $H_{S}$, $H_{T}$, and $G_{S T}$ and then examine the temporal changes of these quantities.

\section{MATHEMATICAL MODEL USED}

The mathematical model we use is that of Maruyama (1970) with Nei's (1975) correction (see also Latter, 1973). In this model, it is assumed that there are $n$ randomly mating subpopulations of effective size $N$, and the immigrants into a subpopulation are a random sample of individuals from the whole population. We consider the infinite allele model of neutral mutation and denote the mutation rate per locus by $u$ and the migration rate by $m$, where $m=n m^{\prime} /(n-1)$ and $\mathrm{Nm}^{\prime}$ is the number of individuals that a given subpopulation exchanges with all others in one generation. Let $J_{0}$ be the probability of identity of two randomly chosen genes from a subpopulation, and $J_{1}$ be the probability of identity of two randomly chosen genes, one from each of two subpopulations. The expected gene diversity (heterozygosity) within subpopulations is given by $H_{S}=1-J_{0}$, whereas the expected gene diversity for the entire population is $H_{T}=1-J_{1}-\left(J_{0}-J_{1}\right) / n$ (Nei, 1975). $G_{S T}$ is defined as $G_{S T}=D_{S T} / H_{T}$, where $D_{S T}=(1-1 / n)\left(J_{0}-J_{1}\right)$. Nei $(1975)$ has shown that the equilibrium values of $J_{0}$ and $J_{1}$ are given by

$J_{0}^{*}=(1-u)^{2}\left[a-(1-m)^{2}(1-u)^{2}\right] /(2 N A)$,

$J_{1}^{*}=b(1-u)^{2} /(2 N A)$,

where $a=(1-m)^{2}+b, b=m(2-m) / n$, and

$$
\begin{aligned}
A= & 1-(1-u)^{2}\left[1+(1-m)^{2}-a /(2 N)\right] \\
& +(1-m)^{2}(1-u)^{4}[1-1 /(2 N)] .
\end{aligned}
$$

Therefore, we can compute the equilibrium values of $H_{S}, H_{T}$, and $G_{S T}$.
In nonequilibrium populations, the values of $J_{0}$ and $J_{1}$ at the $t$ th generations $\left[J_{0}(t)\right.$ and $\left.J_{1}(t)\right]$ are given by

$$
\begin{aligned}
& J_{0}(t)=J_{0}^{*}+c_{1}\left[(1-u)^{2} \lambda_{1}\right]^{t}+c_{2}\left[(1-u)^{2} \lambda_{2}\right]^{t}, \\
& J_{1}(t)=J_{1}^{*}+c_{3}\left[(1-u)^{2} \lambda_{1}\right]^{t}+c_{4}\left[(1-u)^{2} \lambda_{2}\right]^{t},
\end{aligned}
$$

where $c_{1}, c_{2}, c_{3}$, and $c_{4}$ are constants determined by $n, N$, and $m$ as well as by the initial values $J_{0}(0)$ and $J_{1}(0)(\mathrm{Li}, 1976)$. The steady-state rate of approach to equilibrium (steady rate of decay of gene diversity) is $1-\lambda_{1}$, where $\lambda_{1}$ is the dominant eigenvalue. It is given by

$$
\lambda_{1}=\left[(1+a-b)+\sqrt{(1+a-b)^{2}-4 a}\right] / 2,
$$

where $\quad a=(1-m)^{2}[1-1 /(2 N)]$, and $b=$ $m^{2}(2-m) /(2 n N)$. The formula for the second eigenvalue $\lambda_{2}$ is similar to (3) but has a minus sign in front of the square root term. In a panmictic population, $1-\lambda_{1}=1 /(2 N)$ (Wright, 1931).

\section{MIGRATION EFFECTS AT EQUILIBRIUM}

We first examine the effect of migration on the equilibrium values of $H_{S}, H_{T}$, and $G_{S T}$ because the numerical relationships of these values are not well studied. To see the effects of population structure, we consider various combinations of $n, N$, and $m$, and two different total population sizes $\left(n N=100\right.$, and $\left.=10^{5}\right)$. Special attention is given to the case of $N m^{\prime}=1$ because of the importance attributed to the "one migrant per generation" rule in the recent literature. The other migration rates used are of different orders of magnitudes larger or smaller than this, the limits being complete isolation $(m=0)$ and panmixia $(m=1)$.

\section{Degree of genetic differentiation}

Migration has a homogenising role in a subdivided population and its effect can be measured by $F_{S T}$ or $G_{S T}$, the degree of genetic differentiation. Using the infinite island model with two alleles, Wright (1931, 1969) states that the extent of genetic differentiation is "significant" if $F_{S T}\left(=G_{S T}\right)>0.33$ and "fairly extensive" even when $F_{S T}=0.05$ [see also Endler (1977, p. 38) and Kimura and Ohta (1971, p. 122)].

In Wright's infinite island model, genetic variability can be maintained without mutation. In the finite island model, however, we must have mutation to maintain genetic variability. Our preliminary study has shown that if the total population size is $n N=100$, the equilibrium value of $G_{S T}$ is virtually the same for the mutation rates 
ranging from $10^{-7}$ to $10^{-5}$, but in a large population the mutation rate has a noticeable effect. In the following and subsequent computations, we have assumed $u=10^{-6}$ unless otherwise mentioned.

Table 1 shows the values of $H_{S}, H_{T}, G_{S T}$, and $1-\lambda_{1}$ for various values of $m, n$, and $N$. With a given number of subpopulations $(n)$, the equilibrium of $G_{S T}$ depends largely on the product of $N$ and $m^{\prime}$ rather than on either of them separately. For instance, when $N m^{\prime}=1$, the $G_{S T}$ values in the populations with $n N=100$ and $n N=10^{5}$ are 0.0555 and 0.0575 , respectively, for $n=2$, and $0 \cdot 1449$ and $0 \cdot 1671$ for $n=10$. However, $G_{S T}$ is dependent on the number of subpopulations. For example, in the case of $n N=10^{5}$ and $N m^{\prime}=1, G_{S T}$ is 0.0575 for $n=2,0 \cdot 1671$ for $n=10$, and $0 \cdot 1964$ for $n=100$ (table 1$). G_{S T}$ is always smaller than that $[1 /(4 N m+1)]$ expected for the infinite island model with the same amount of migration. When there are only two subpopulations (either large or small), the expected amount of differentiation with
$N m^{\prime}=1$ is about the same as that for the infinite island model with $\mathrm{Nm}^{\prime}=4 \cdot 2$.

\section{Amount of gene diversity}

From the point of view of genetic conservation, we are interested not only in the degrees of differentiation between the subpopulations but in the actual amount of genetic variability maintained. Gene diversities, both $H_{S}$ and $H_{T}$, can be obtained by using (1).

When the total population size is small, i.e., $n N=100$, the gene diversity is close to zero both within the subpopulations and in the total population irrespective of the values of $n$ and $m^{\prime}$ (table 1 ). Only when the subpopulations are virtually isolated from each other does the total gene diversity at equilibrium become substantial. This occurs because different mutations accumulate in different populations in this case. The pattern of population subdivision and migration rate become

Table 1 Equilibrium values of $H_{S}, H_{T}, G_{S T}$, and $1-\lambda_{1}$. The mutation rate is assumed to be $u=10^{-6}$

\begin{tabular}{|c|c|c|c|c|c|c|}
\hline \multirow[b]{2}{*}{$n$} & \multirow[b]{2}{*}{$N$} & \multirow[b]{2}{*}{$N m^{\prime}$} & \multicolumn{4}{|c|}{ Equilibrium values } \\
\hline & & & $H_{S}$ & $H_{T}$ & $G_{S T}$ & $1-\lambda_{1}$ \\
\hline \multirow[t]{7}{*}{10} & 10 & 0 & 0.0001 & 0.9001 & 0.999 & - \\
\hline & & 0.001 & 0.0004 & 0.0747 & 0.9950 & $2 \cdot 2 \times 10^{-5}$ \\
\hline & & 0.01 & 0.0004 & 0.0084 & 0.9528 & $2.1 \times 10^{-4}$ \\
\hline & & $0 \cdot 1$ & 0.0004 & 0.0012 & 0.6657 & $1.5 \times 10^{-3}$ \\
\hline & & 1 & 0.0004 & 0.0005 & $0 \cdot 1449$ & $4.2 \times 10^{-3}$ \\
\hline & & 2 & 0.0004 & 0.0004 & 0.0644 & $4.6 \times 10^{-3}$ \\
\hline & & Panmixia & 0.0004 & 0.0004 & 0.0000 & $5.0 \times 10^{-3}$ \\
\hline \multirow[t]{2}{*}{4} & 25 & 0.1 & 0.0004 & 0.0010 & 0.5284 & $1.8 \times 10^{-3}$ \\
\hline & & 1 & 0.0004 & 0.0005 & $0 \cdot 1146$ & $4.4 \times 10^{-3}$ \\
\hline \multirow[t]{2}{*}{2} & 50 & $0 \cdot 1$ & 0.0004 & 0.0007 & 0.3831 & $2.6 \times 10^{-3}$ \\
\hline & & 1 & 0.0004 & 0.0004 & 0.0555 & $4.7 \times 10^{-3}$ \\
\hline \multirow[t]{8}{*}{100} & 1,000 & 0 & 0.0040 & 0.9901 & 0.9960 & - \\
\hline & & 0.001 & 0.0079 & 0.9804 & 0.9919 & $2.0 \times 10^{-8}$ \\
\hline & & 0.01 & 0.0385 & 0.9039 & 0.9574 & $1.9 \times 10^{-7}$ \\
\hline & & $0 \cdot 1$ & $0 \cdot 1687$ & 0.4781 & 0.7081 & $1.4 \times 10^{-6}$ \\
\hline & & 1 & 0.2671 & 0.3323 & $0 \cdot 1964$ & $4.0 \times 10^{-6}$ \\
\hline & & 10 & 0.2838 & 0.2906 & 0.0235 & $4.9 \times 10^{-6}$ \\
\hline & & 100 & 0.2856 & 0.2862 & 0.0021 & $5.0 \times 10^{-6}$ \\
\hline & & Panmixia & 0.2857 & 0.2857 & 0.0000 & $5.0 \times 10^{-6}$ \\
\hline \multirow[t]{7}{*}{10} & 10,000 & 0 & 0.0385 & 0.9038 & 0.9575 & - \\
\hline & & 0.001 & 0.0419 & 0.8957 & 0.9532 & $2.0 \times 10^{-8}$ \\
\hline & & 0.01 & 0.0679 & 0.8305 & 0.9182 & $1.9 \times 10^{-7}$ \\
\hline & & $0 \cdot 1$ & $0 \cdot 1866$ & 0.5335 & 0.6503 & $1.5 \times 10^{-6}$ \\
\hline & & 1 & 0.2702 & 0.3245 & $0 \cdot 1671$ & $4.1 \times 10^{-6}$ \\
\hline & & 10 & 0.2842 & 0.2899 & 0.0198 & $4.9 \times 10^{-6}$ \\
\hline & & 100 & 0.2856 & 0.2862 & 0.0019 & $4.9 \times 10^{-6}$ \\
\hline \multirow[t]{2}{*}{2} & 50,000 & $0 \cdot 1$ & $0 \cdot 2501$ & $0 \cdot 3750$ & 0.3333 & $2.6 \times 10^{-6}$ \\
\hline & & 1 & 0.2808 & 0.2980 & 0.0575 & $4.7 \times 10^{-6}$ \\
\hline
\end{tabular}

$\mathrm{Nm}^{\prime}$ is the number of migrants per subpopulation in one generation.

$H_{S}$ and $H_{T}$ are the gene diversities within subpopulations and in the total population.

$G_{S T}$ is the degree of subpopulation differentiation.

$1-\lambda_{1}$ is the rate of decay of gene diversity at steady state. 
important when the total population size is large, say, $n N=10^{5}$. If a population of this size is completely panmictic, the expected gene diversity for the infinite-allele model is 0.286 (both $H_{S}$ and $H_{T}$ ). When $\mathrm{Nm}^{\prime}>1, H_{S}$ and $H_{T}$ are similar to those for panmixia, as the previous authors have noted. However, as $\mathrm{Nm}^{\prime}$ decreases, $H_{S}$ gradually decreases and $H_{T}$ increases. Note that $N m^{\prime}=0 \cdot 1$ corresponds to the case where one individual migrates every 10 generations in each subpopulation. Note also that when $n N=100, H_{S}$ is the same as that of panmixia even when $N m^{\prime}=0 \cdot 1$, and that generally this amount of migration is enough to keep $H_{S}$ and $H_{T}$ halfway between those for panmixia and complete isolation.

\section{Decay of genetic variation at steady state}

In a finite population, gene diversity declines in every generation by the effect of genetic drift until this decrease is balanced by the increment due to mutation. In a population of a given size, this rate of decay of gene diversity will eventually reach a steady state value given by (3). We next examine how the pattern of population subdivision and migration affect this rate.

Although the rate of decay is largely determined by $\mathrm{Nm}^{\prime}$, it also depends on the number of subpopulations $(n)$. This effect can be examined by asking what is the critical migration rate that is sufficient to cause the subdivided population to behave as if it were panmictic. Of course the $\lambda$, value for a subdivded population is always larger than that $\left(\lambda_{p}\right)$ of a panmictic population, but if $1-\lambda_{1}$ reaches $0.95\left(1-\lambda_{p}\right)$, we may regard that the population is "nearly panmictic." With this criterion, we can investigate the number of migrants required for a population to reach panmixia.

It appears that the number of migrants per generation required to approximate panmixia settles within a fairly narrow range, $0 \cdot 9-4 \cdot 7$, for the combinations of $n$ varying from 2 to 128 and $N$ from 10 to 40,960 (fig. 1 ). When $N \geqq 40$, the critical $N^{\prime}$ value is determined mainly by the number of subpopulations $(n)$. When $N$ ranges from 40 to 40,960 , the $\mathrm{Nm}^{\prime}$ values are within 1.15 1.27 for $n=2$, within $2 \cdot 44-2 \cdot 72$ for $n=4$, within 3.19-3.72 for $n=8$, and within $3 \cdot 61-4 \cdot 60$ for $n=$ $16 \sim 128$ (fig. 1). The critical $\mathrm{Nm}^{\prime}$ values increase with both $n$ and $N$, but for a fixed total population size $(n N)$, the maximal $N m^{\prime}$ is obtained for intermediate values of $n$ and $N$.

If we use a more stringent criterion of $\left(1-\lambda_{1}\right)=$ $0.99\left(1-\lambda_{p}\right)$, the critical $N m^{\prime}$ values range from $2 \cdot 4$ to $24 \cdot 1$ for the same set of $n$ and $N$.

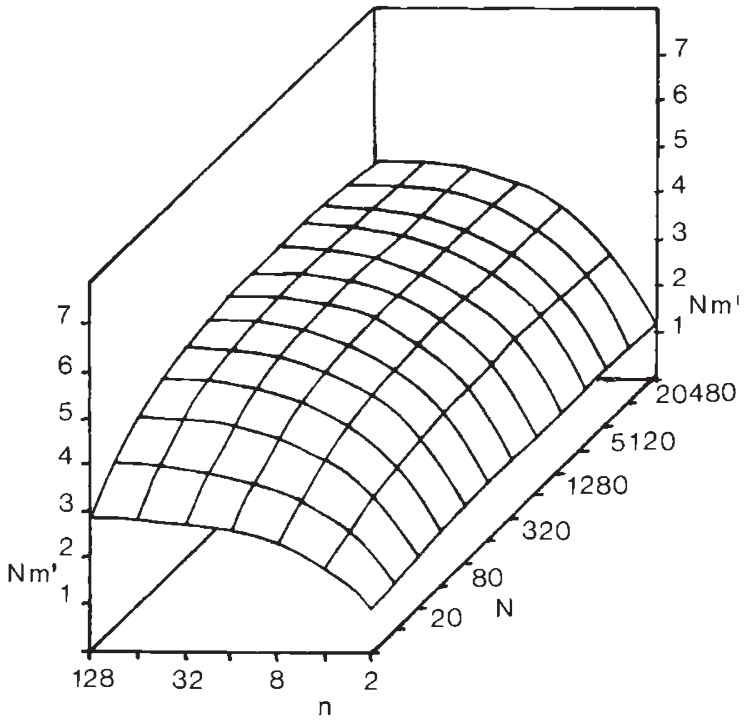

Figure 1 The number of migrants $\left(\mathrm{Nm}^{\prime}\right)$ per subpopulation in one generation that results in a rate of decay of heterozygosity departing only 5 per cent from that rate for a panmictic population of size $n N$.

\section{NUMBER OF GENERATIONS NEEDED TO ATTAIN EQUILIBRIA}

Applying the equilibrium theory to natural populations may not always be justified, and if applied to management of genetic resources, there is a risk of unjustified actions. Therefore, it is important to examine the transition from the initial conditions to the equilibrium. Here, we examine how long it takes for each of $H_{S}, H_{T}$, and $G_{S T}$ to reach the equilibrium values approximately. Theoretically, the quantities we examine here approach the equilibrium values only asymptotically, and the time becomes infinite. (In reality, the values have a distribution with a non-zero variance and there is a certain probability that the population reaches the equilibrium. We discuss here only the expected values.) We, therefore, use the same approach as the above and examine how much time is required for a value departing only 5 per cent from the equilibrium value to be reached. The values of $H_{S}, H_{T}$, and $G_{S T}$ in the transient state can be obtained from equations (2).

Our preliminary studies have shown that the time required is quite insensitive to the initial values of $J_{0}$ and $J_{1}$. Therefore, the following conclusions seem to be quite general as long as the mutation rate is between $10^{-7}$ and $10^{-5}$.

The first quantity to reach its stable value is the rate of decay of the total gene diversity. In 
Table 2 Numbers of generations required for $H_{S}, H_{T}$, and $G_{S T}$ to reach a value departing only 5 per cent from the equilibrium value

\begin{tabular}{|c|c|c|c|c|c|}
\hline \multirow[b]{2}{*}{$n$} & \multirow[b]{2}{*}{$N$} & \multirow[b]{2}{*}{$N m^{\prime}$} & \multicolumn{3}{|c|}{$\begin{array}{l}\text { Number of generations } \\
\text { required for }\end{array}$} \\
\hline & & & $H_{S}$ & $H_{T}$ & $G_{S T}$ \\
\hline \multirow[t]{5}{*}{10} & 10 & 0 & 40 & $10^{6}$ & 60 \\
\hline & & $0 \cdot 1$ & 600 & $10^{3}$ & 30 \\
\hline & & 1 & 500 & 500 & 9 \\
\hline & & 2 & 400 & 500 & 6 \\
\hline & & Panmixia & 400 & 400 & 0 \\
\hline \multirow[t]{3}{*}{4} & 25 & 0 & 100 & $10^{6}$ & 100 \\
\hline & & $0 \cdot 1$ & 600 & $10^{3}$ & 80 \\
\hline & & 1 & 400 & 500 & 10 \\
\hline \multirow[t]{5}{*}{100} & 1,000 & 0 & $4 \times 10^{3}$ & $3 \times 10^{6}$ & $6 \times 10^{3}$ \\
\hline & & $0 \cdot 1$ & $2 \times 10^{3}$ & $4 \times 10^{5}$ & $4 \times 10^{3}$ \\
\hline & & 1 & $2 \times 10^{5}$ & $2 \times 10^{5}$ & $10^{3}$ \\
\hline & & 100 & $2 \times 10^{5}$ & $2 \times 10^{5}$ & 9 \\
\hline & & Panmixia & $2 \times 10^{5}$ & $2 \times 10^{5}$ & 0 \\
\hline \multirow[t]{3}{*}{10} & 10,000 & 0 & $4 \times 10^{4}$ & $2 \times 10^{6}$ & $7 \times 10^{4}$ \\
\hline & & $0 \cdot 1$ & $2 \times 10^{4}$ & $2 \times 10^{5}$ & $4 \times 10^{4}$ \\
\hline & & 1 & $2 \times 10^{5}$ & $2 \times 10^{5}$ & $10^{4}$ \\
\hline
\end{tabular}

table 3 , the number of generations required for the ratio of gene diversities in two successive generations to reach the same order of magnitude as the steady state value is given. Unless the migration rate is very low $\left(\mathrm{Nm}^{\prime} \leqq 0.01\right)$, the rate of decay of $H_{T}$ almost immediately reaches the steady state value (table 3 ).

Table 3 Numbers of generations required for the rate of decay of heterozygosity to reach the steady state value (to the order of magnitude). No mutation is assumed

\begin{tabular}{|c|c|c|c|c|}
\hline \multirow[b]{2}{*}{$n$} & \multirow[b]{2}{*}{$N$} & \multirow[b]{2}{*}{$N m^{\prime}$} & \multicolumn{2}{|c|}{$\begin{array}{l}\text { Number of generations } \\
\text { required }\end{array}$} \\
\hline & & & $\begin{array}{l}\text { Within } \\
\text { subpopulations }\end{array}$ & $\begin{array}{l}\text { In total } \\
\text { population }\end{array}$ \\
\hline \multirow[t]{5}{*}{10} & 10 & 0.001 & 200 & 50 \\
\hline & & 0.01 & 140 & 36 \\
\hline & & $0 \cdot 1$ & 40 & 0 \\
\hline & & 1 & 8 & 0 \\
\hline & & 2 & 5 & 0 \\
\hline \multirow{6}{*}{100} & 1,000 & 0.001 & 20,000 & 8,000 \\
\hline & & 0.01 & 20,000 & 4,000 \\
\hline & & 0.1 & 7,000 & 0 \\
\hline & & 1 & 1,000 & 0 \\
\hline & & 10 & 200 & 0 \\
\hline & & 100 & 21 & 0 \\
\hline \multirow[t]{6}{*}{10} & 1,000 & 0.001 & 20,000 & 8,000 \\
\hline & & 0.01 & 10,000 & 3,000 \\
\hline & & $0 \cdot 1$ & 4,000 & 0 \\
\hline & & 1 & 900 & 0 \\
\hline & & 10 & 200 & 0 \\
\hline & & 100 & 9 & 0 \\
\hline \multirow[t]{3}{*}{2} & 1,000 & 0.001 & 10,000 & 8,000 \\
\hline & & 0.01 & 9,000 & 2,000 \\
\hline & & $0 \cdot 1$ & 0 & 0 \\
\hline
\end{tabular}

It takes a considerably long time for $G_{S T}$ and the rate of decay of $H_{S}$ to attain their steady state values (tables 2,3). For a given total population size, the within-subpopulation rate of decay reaches the steady state value faster when there are few subpopulations than when there are many. By contrast, $G_{S T}$ has the opposite tendency.

The gene diversities, $H_{S}$ and $H_{T}$, reach their equilibrium values much more slowly than $G_{S T}$ (table 2). When the migration rate is high, these two parameters approach the equilibrium values approximately at the same time. This is expected on the basis of our earlier finding that the migration rates with $\mathrm{Nm}^{\prime}>1$ (with the scale used here) keep the populations close to panmixia. With small migration rates, $H_{S}$ will reach the equilibrium faster when there are many small subpopulations, whereas $H_{T}$ has the opposite tendency (table 2).

One implication of these results is that in natural populations $G_{S T}$ is more likely to be close to its equilibrium value than $H_{S}$ and $H_{T}$. The quick approach to equilibrium of $G_{S T}$ has previously been noticed by Nei et al. (1977) and Crow and Aoki (1984).

\section{MIGRATION EFFECTS IN TRANSIENT STATES}

We have seen that a small amount of migration $\left(\mathrm{Nm}^{\prime}>1\right)$ is usually sufficient to make a finite subdivided population in equilibrium to behave just like a panmictic population. We have also seen that the time required for this to occur is generally very long, at least from the perspective of shortterm genetic management. Therefore, it is necessary to examine the behaviours of $G_{S T}(t), H_{S}(t)$, and $H_{T}(t)$ in transient states. We have carried out such computations using (2) and several initial conditions. The total population sizes used are, again, $n N=100$ and $n N=10^{5}$. We have also used the same patterns of population subdivision as before (table 1 ). The migration rates used are $N m^{\prime}=0,0 \cdot 1,1 \cdot 0$ and $(n-1) N / n$, the last one corresponding to the case of panmixia with $m=1$. In addition, we have included the case with $m=0 \cdot 15$.

\section{Behaviour of $G_{S T}$}

We consider a situation where all subpopulations are initially identical to each other with $J_{0}(0)=$ $J_{1}(0)=0 \cdot 5$. $G_{S T}$ will eventually reach the equilibrium states (table 1), and we have examined the trajectory of $G_{S T}(t)$ from the initial value to the equilibrium value. 
In small populations ( $n N=100)$, the differentiation of subpopulations starts immediately, except when $m=0.15$, which is enough to keep the entire population as if it were panmictic (fig. 2). With smaller migration rates, the trajectories of $G_{S T}$ follow that of complete isolation in the early generations and then branch off from this curve in the order of decreasing migration rates (fig. 2(a-c)). In large populations $\left(n N=10^{5}\right)$, it takes a longer period of time before the differences in $G_{S T}$ among different $\mathrm{Nm}^{\prime}$ 's become apparent. This difference in the behaviour of $G_{S T}$ between small and large populations occurs because the rate of decay of gene diversity is higher in small populations than in large populations, and the balance between mutation and genetic drift is attained more quickly. This seems to be due to the fact that in the early generations the effect of genetic drift dominates and $G_{S T}$ overshoots the equilibrium level. In the later generations, however, $G_{S T}$ gradually declines because of the joint effect of mutation and migration.

\section{Behaviour of gene diversities}

Similar results are obtained for the gene diversities, which are here expressed in terms of gene iden-

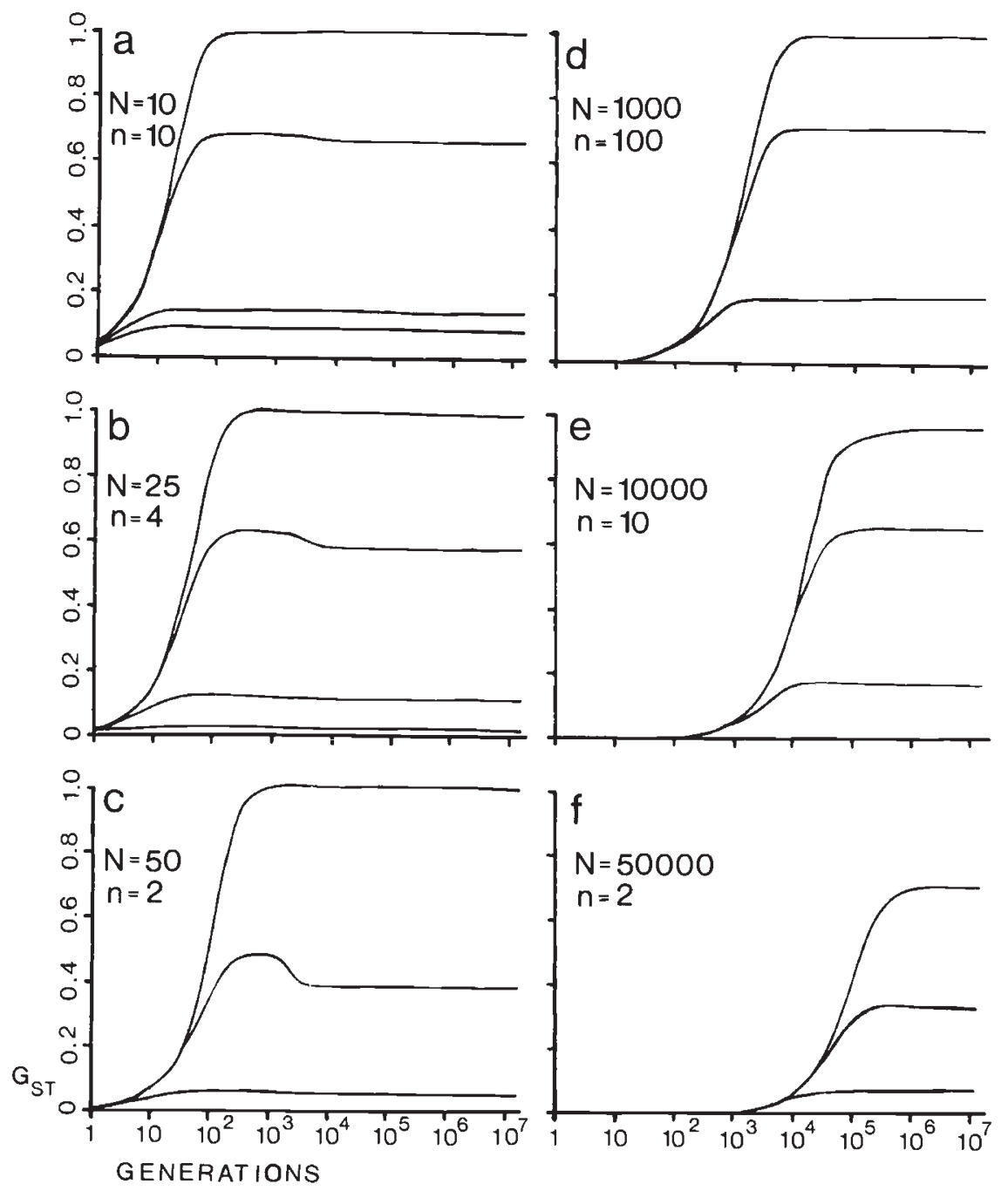

Figure 2. Population differentiation $\left(G_{S T}\right)$ for various combinations of the number of subpopulations $(n)$ and subpopulation size $(N)$. The initial gene diversity is 0.5 both within and between subpopulations. The curves from top to bottom are: complete isolation $(m=0)$, one migrant in every tenth generation $\left(N m^{\prime}=0 \cdot 1\right)$, one migrant in every generation $\left(N m^{\prime}=1\right)$, and a migration rate $m=0.15$ (not distinguishable from the zero line in figs c-f). 
tifies, $J_{0}=1-H_{S}$ and $J_{T}=1-H_{T}$. Let us first consider the case where the subpopulations are initially identical to each other with $J_{0}(0)=J_{1}(0)=$ $0 \cdot 5$. In a small population $(n N=100)$, each subpopulation eventually becomes monomorphic ( $\mathrm{cf}$. table 1), and the time required for this was already examined (table 2 ). When there are only two subpopulations, the trajectories of $J_{0}$ under panmixia and complete isolation are very close to each other. When the population of the same size is subdivided into many subpopulations, the subpopulations go to fixation more rapidly (fig. 3(a-c)). With some migration between the subpopulations, the situ- ation is similar to that seen above with $G_{S T}$; the trajectory of $H_{S}$ in the early generations is similar to that of the case of complete isolation. In the later generations, however, it branches off from the latter curve and joins that of a panmictic population. Migration rate with $N m^{\prime}=1$ keeps the trajectory of $J_{0}$ close to that of panmixia, whereas $\mathrm{Nm}^{\prime}=0 \cdot 1$ keeps the population close to the case of complete isolation for most of the time.

The total gene identity $J_{T}$ also increases, although more slowly than $J_{0}$ and much more slowly with low migration (fig. $3(\mathrm{a}-\mathrm{c})$; note the logarithmic time scale). In the case of $n N=100$,
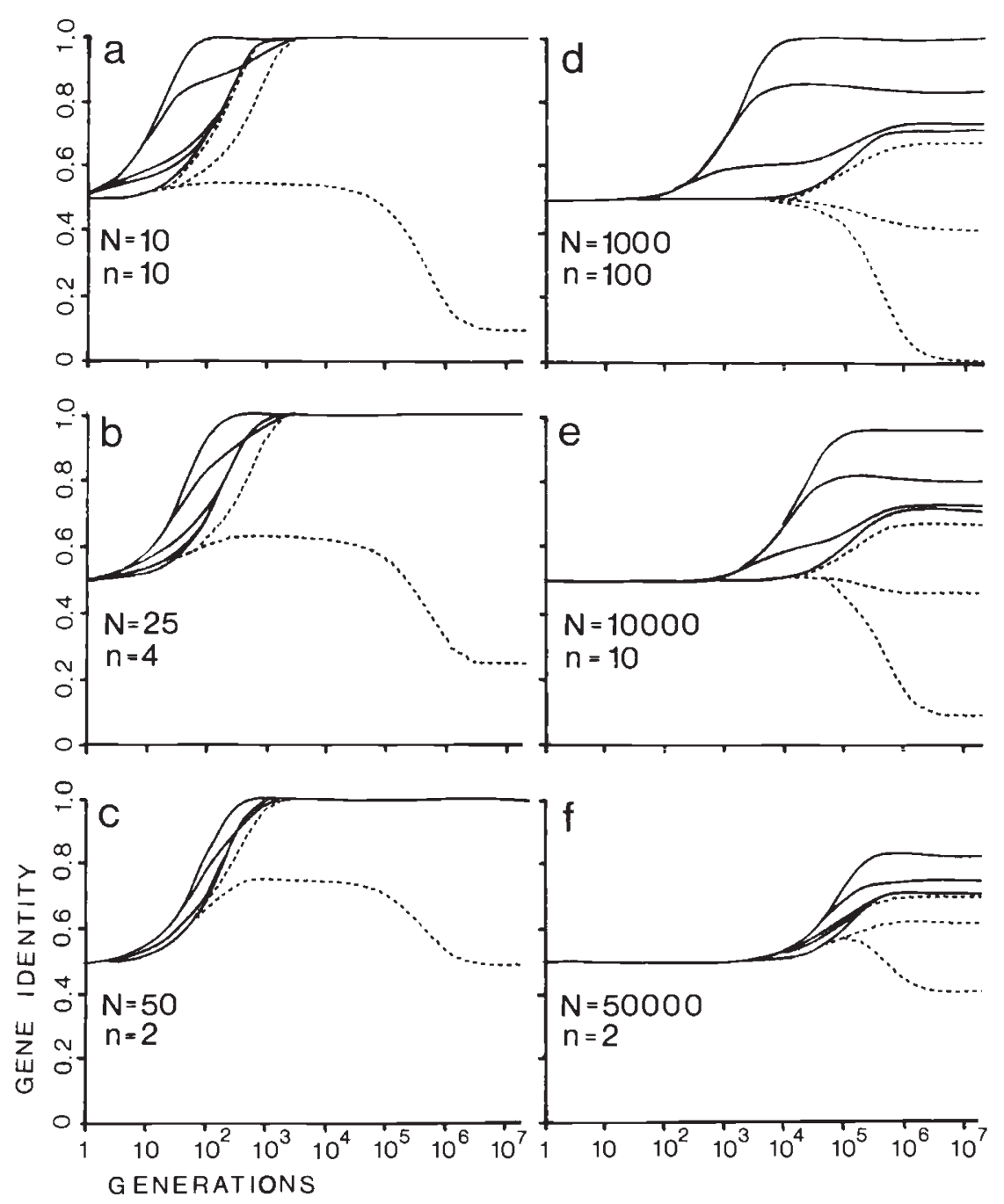

Figure 3 Gene identity within subpopulations (solid lines) and in the total population (broken lines). The migration rates and initial gene identities are the same as in fig. 2, except that the case of complete panmixia has been added here. Complete isolation is represented by the topmost curve for within-subpopulation gene identity and by the bottom line for the total gene identity. In each of the cases given in the figure, there are nine curves, but all curves are not distinguishable when migration rates are high. Note that $H_{S}$ and $H_{T}$ are identical in the case of panmixia. 
$J_{T}$ eventually becomes close to 1 except for the case of $m=0$. In the latter case, $J_{T}$ decreases in the later generations because different mutations accumulate in different subpopulations. This decrease of $J_{T}$ also requires transient polymorphisms in the subpopulations, but in small subpopulations such polymorphisms have a short duration, and the expectation of $J_{0}$ is close to one.

The results for a large population $\left(n N=10^{5}\right)$ are similar to those above, except that there is a time lag during which the gene identity remains at the initial level (fig. 3(d-f)). The equilibrium values of $J_{0}$ and $J_{T}$ are clearly smaller than one, and the differences between the curves representing different migration rates are larger than those in small populations. Initially, $J_{0}$ in the populations with $N m^{\prime}=1$, or $=0 \cdot 1$, follows the trajectory of complete isolation, but in general the migration rate with $N m^{\prime}=1$ keeps the value of $J_{0}$ very close to that of a panmictic population. The same is true for $J_{T}$. With $N m^{\prime}=0 \cdot 1$, the early behaviours of $J_{0}$ and $J_{T}$ resemble the situation for the case of completely isolated subpopulations, although the equilibrium values are intermediate between the cases of complete isolation and panmixia (table 1).

To examine the transient values of $J_{0}$ and $J_{T}$ for the case where the subpopulations are not initially identical, we computed their trajectories for several cases with $J_{0}(0) \neq J_{1}(0)$ using the same migration rates as above. If the subpopulations are completely isolated, $J_{0}$ is, of course, expected to increase monotonically toward its equilibrium value. In a panmictic population, the allele frequency differences between the subdivisions disappear in the first generation, after which $J_{0}\left(=J_{T}\right)$ increases toward the equilibrium value. With restricted migration, the trajectories are placed between these two extremes, and their behaviours depend on the initial conditions and the migration rate (fig. 4). In small populations, $J_{0}$ initially decreases because of mixing of different gene pools, and then starts to increase toward the equilibrium value. During this period of the first 10 to 100 generations, large differences exist in the values of $J_{0}$ depending on the migration rate, whereas the differences in $J_{T}$ become apparent after that early period. Only complete isolation keeps $J_{T}$ continuously at a low level. With the migration rates used here, the most conspicuous difference in the $J_{0}$ values seems to be between the migration rates $N m^{\prime}=0 \cdot 1$ and $N m^{\prime}=1$, at least in small populations (fig. $4(\mathrm{a}-\mathrm{b})$ ).

Finally, note that while a low migration rate $\left(N m^{\prime}=0 \cdot 1\right)$ makes the subpopulations initially

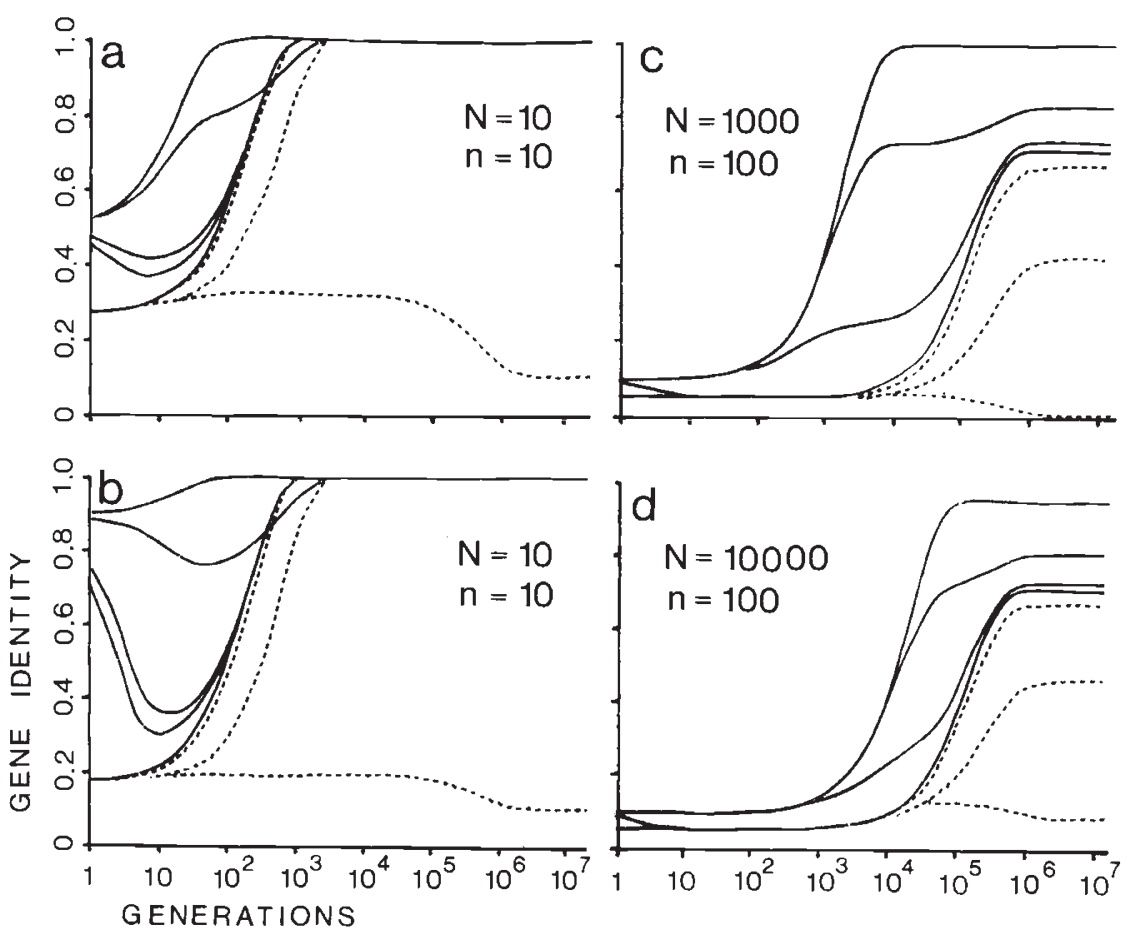

Figure 4 Gene identities as in fig. 3 , but the initial conditions vary: (a) $J_{0}(0)=0 \cdot 5$ and $J_{1}(0)=0 \cdot 25 ;(\mathrm{b}) J_{0}(0)=0 \cdot 9$ and $J_{1}(0)=0 \cdot 1$; (c) and (d) $J_{0}(0)=0.1$ and $J_{1}(0)=0.05$. 
behave as if they were completely isolated and leads to a rapid increase in $J_{0}$, it helps to maintain the total gene diversity $\left(H_{T}\right)$ at a higher level than that of the cases of higher migration rates. For this reason, the within-subpopulation gene identity $\left(J_{0}\right)$ for such a low migration rate is also expected to reach its equilibrium later than for the case of high migration rates. This is seen from the curves which cross over in fig. $3(\mathrm{a}-\mathrm{c})$ and $4(\mathrm{a}-\mathrm{b})$, and from the $J_{0}$ values in table 2 .

\section{DISCUSSION}

In the applications of population genetics theory to the management of endangered species, the goal seems to have been to build up a population which functions as a panmictic unit. Several authors have suggested that exchanging a very small proportion of individuals between populations of a reasonable size will establish effective panmixia. Furthermore, it has been suggested that the induced gene flow results in increased effective population size, keeping the composition of alleles similar (e.g., Frankel, 1983; Chambers, 1983).

One basic question here is whether one should try to maintain the maximal variability within the entire species (population) or within single subpopulations. It is evident that the expected transient values of $H_{T}$ and $H_{S}$ are different from each other. With very low migration, homozygosity increases within the subpopulations, but the subpopulations also differentiate from each other in the long run and the total gene diversity remains high. In practice, one has to make a compromise between these two aims, and the decision should also depend on whether isolated subpopulations can tolerate homozygosity caused by their small size. If the total population size is large, these effects become apparent only after a long time, but in small populations the changes in gene diversity take place immediately.

There are also differences in long-term consequences. Making the entire species panmictic helps to maintain the within-population gene diversity at a higher level in early generations, but this is followed by a more rapid loss of total variation. For instance, the example in fig. 4(b) shows that when $N m^{\prime}=1, H_{S}$ is much greater than when $N m^{\prime}=0 \cdot 1$ for approximately 200 generations. After this period, the expected $H_{S}$ is the same under both migration rates, but the total gene diversity within the species is expected to be larger with the lower migration rate; $H_{T}=0.2$ for $N m^{\prime}=1$ and $H_{T}=0.5$ for $N m^{\prime}=0 \cdot 1$. The time period of
200 generations is, of course, generally very long for such small populations, at least to be maintained in stable numbers, but the example illustrates the duality of the problem.

Earlier discussions of the effect of migration have largely been based on Wright's (1931) infinite island model. Lewontin (1974:213) concluded on the basis of allele frequency distributions that $N m^{\prime}=1$ is enough to keep the allele frequency differences small between subpopulations. Allendorf (1983) suggests that the ideal amount of exchange among subpopulations is, on average, one migrant per generation, since this amount of migration is sufficient to avoid the loss of alleles. He further hints that $N m^{\prime}=1$ should ensure that all genetic variation of a species is present in all subpopulations and would also allow genetic differentiation in response to local selection pressures. In another context, Allendorf and Phelps (1981) point out that the predictive power of the principle of low migration is very weak at any single point of time. In general, the rule of "one migrant per generation" has been widely accepted as a guideline in population management (e.g., Frankel and Soulé, 1981).

Our results give limited support for these conclusions in that the transient values of both the gene diversities and $G_{S T}$ show a large difference between the migration rates with $N m^{\prime}=0 \cdot 1$ and $N m^{\prime}=1$. The former makes the population to behave as if the subpopulations were completely isolated, and the latter gives results close to panmixia. But the results also show that the transient values depend not only on migration rates but also on the subdivision pattern, and perhaps on the interaction between the two. The $G_{S T}$ values associated with certain migration rate, say, $\mathrm{Nm}^{\prime}=$ 1 , are highly dependent on the number of subpopulations. Takahata's (1983) computations also show the same result for small $n$. Li (1976) consider the question in terms of gene identity between subpopulations and points out that the effect of exchanging individuals between two subpopulations is smaller when the number of subpopulations increases, and hence the gene identity is smaller. The larger $n$ is, the more does the genetic composition of the immigrants differ from that of the nonimmigrant individuals.

We want to stress that all results presented here concern the expected values. In finite populations, the stochastic variances of $H_{S}, H_{T}$, and $G_{S T}$ are very large unless they are computed from a large number of loci (Li and Nei, 1975; Nei et al., 1977; Takahata, 1983). Our computer simulations have also indicated that the mean values are not very 
informative for predicting the behaviour of $H_{S}, H_{T}$, and $G_{S T}$ for individual loci. For example, the distribution of gene diversities is rather flat already after 10 generations when $n=N=$ $10, N m^{\prime}=1$ and $J_{0}(0)=J_{1}(0)=0 \cdot 5$. The variances of $J_{0}, J_{1}$, and $G_{S T}$ at equilibrium have been recently discussed by Golding and Strobeck (1983) and Takahata (1983). Some studies on the transient-state variances of $H_{S}, H_{T}$, and $G_{S T}$ have also been conducted (Nei et al., 1977). It is important to take into account the non-equilibrium behaviour of these quantities not only in genetic management but in interpreting any results from small natural populations.

In practical situations, the problem is complicated by fluctuating population sizes, splitting and extinction of subpopulations, etc. At the moment, we have to conclude that the theoretical basis of the genetic management of small, endangered populations is not well understood, and selecting any single guideline like the "one migrant per generation" rule is not theoretically well justified.

Acknowledgements This study has been supported by grants from the National Institutes of Health, the National Science Foundation and the Fulbright Foundation. The invaluable comments and help of P. Pamilo and R. Väinöla are gratefully acknowledged.

\section{REFERENCES}

ALlENDORF, F. W. 1983. Isolation, gene flow, and genetic differentiation among populations. Schonewald-Cox, C. M., Chambers, S. M., MacBryde, B. and Thomas, L. (eds.) In Genetics and Conservation, Benjamin/Cummings, pp. 51-65.

ALLENDORF, F. W. AND PHELPS, S. R. 1981. Use of allelic frequencies to describe population structure. Can. J. Fish. Aquat. Sci. $38,1507-1514$

CHAMBERS, S. M. 1983. Genetics principles for managers, Schonewald-Cox, C. M., Chambers, S. M., MacBryde, B. and Thomas, L. (eds.) In Genetics and Conservation, Benjamin/Cummings, pp. 15-146.

CROW, J. F. AND AOKI, K. 1984. Group selection for a polygenic behavioral trait: Estimating the degree of population subdivision. Proc. Natl. Acad. Sci. USA, 81, 6073-6077.
ENDLER, J. A. 1977. Geographic Variation, Speciation, and Clines, Princeton University Press, Princeton, N.J., U.S.A.

FRANKEL, O. H. 1983. The place of management in conservation, Schonewald, C. M., Chambers, S. M., MacBryde, B. (eds.) In Genetics and Conservation, Benjamin/Cummings, pp. 114.

FRANKEL, O. H. AND SOULÉ. 1981. Conservation and Evolution. Cambridge University Press, Cambridge.

FRANKLIN, 1. R. 1980. Evolutionary change in small populations, Soulé, M. and Wilcox, B. (eds.) In Conservation Biology: An Evolutionary-Ecological Perspective, Sinauer Associates, Sunderland, MA, U.S.A., pp. 135-149.

GOLDING, G. B. AND STROBECK, C. 1983. Variance and covariance of homozygosity in a structured population. Genetics, 104, 513-529.

KIMURA, M. AND MARUYAMA, T. 1971. Pattern of neutral polymorphism in a geographically structured population. Genet. Res., Camb., 18, 125-131.

KIMURA, M. AND OHTA, T. 1971. Theoretical Aspects of Population Genetics, Princeton University Press, Princeton, N.J., U.S.A.

LATTER, B. D. H. 1973. The island model of population differentiation: a general solution. Genetics, 73, 147-157.

LEWONTIN, R. C. 1974. The Genetic Basis of Evolutionary Change, Columbia University Press, New York.

LI, W.-H. 1976. Effects of migration on genetic distance. Amer. Natur., 110, 841-847.

LI, W.-H. AND NEI, M. 1975. Drift variances of heterozygosity and genetic distance in transient states. Genet. Res., Camb., 25, 229-248.

MARUYAMA, T. 1970. Effective number of alleles in a subdivided population. Theor. Popul. Biol., 1, 273-306.

NEI, M. 1973. Analysis of gene diversity in subdivided populations. Proc. Natl. Acad. Sci. USA, 70, 3321-3323.

NEI, M. 1975. Molecular Population Genetics and Evolution. North-Holland, Amsterdam.

NEI, M. AND FELDMAN, M. W. 1972. Identity of genes by descent within and between populations under mutation and migration pressures. Theor. Popul. Biol., 3, 460-465.

NEI, M., CHAKRAVARTI, A. AND TATENO, Y. 1977. Mean and variance of $F_{S T}$ in a finite number of incompletely isolated populations. Theor. Popul. Biol., 11, 291-306.

RALLS, K., BALLOU, J. AND BROWNELL, R. L. 1983. Genetic diversity in California sea otters: theoretical considerations and management implications. Biol. Conserv., 25, 209-232.

TAKAHATA, N. 1983. Gene identity and genetic differentiation of populations in the finite island model. Genetics, 104, 497512.

WRIGHT, S. 1931. Evolution in Mendelian populations. Genetics, $16,97-159$.

WRIGHT, S. 1951. The genetical structure of populations. Annals of Eugenics, 15, 323-354.

WRIGHT, S. 1969. Evolution and the Genetics of Populations, Vol. 2, The Theory of Gene Frequencies, University of Chicago Press, Chicago, IL, U.S.A. 